\title{
Study on the fretting fatigue behavior of alloy ZSGH4169 at different temperatures
}

zeyu xing ${ }^{1}$, Hongjian Zhang ${ }^{2}$, HAITAO CUI ${ }^{2}$, ziqiang $y^{2}{ }^{2}$, xiaodong yan ${ }^{2}$, and WEIDONG $\mathrm{WEN}^{2}$

${ }^{1}$ Affiliation not available

${ }^{2}$ Nanjing University of Aeronautics and Astronautics

November 16, 2020

\begin{abstract}
In this paper, nickel-based alloy ZSGH4169 was used as the test material and the fretting fatigue test of standard test pieces was carried out to study the variation rule of its fretting fatigue life under different temperatures and loads. The results show that the fretting fatigue life of ZSGH4169 decreases with the increase of temperature and load. Through fracture analysis under different working conditions, it can be seen that ZSGH4169 fretting fatigue failure form is basically the same, the crack expands in the form of transgranular fracture propagation. Temperature and load have significant influence on the depth of fretting fatigue crack initiation zone and the fatigue band spacing in the propagation zone, which affects its fretting fatigue life. According to the analysis of crack initiation and propagation path, the crack initiation angle of fretting fatigue crack is mainly affected by temperature. With the increase of temperature, the crack initiation angle increases, however the load has little influence on the crack initiation angle.
\end{abstract}

\section{Hosted file}

Study on the fretting fatigue behavior of alloy ZSGH4169 at different temperatures.pdf available at https://authorea.com/users/376233/articles/493274-study-on-the-frettingfatigue-behavior-of-alloy-zsgh4169-at-different-temperatures

\section{Hosted file}

figure1.pdf available at https://authorea.com/users/376233/articles/493274-study-on-thefretting-fatigue-behavior-of-alloy-zsgh4169-at-different-temperatures

\section{Hosted file}

figure2.pdf available at https://authorea.com/users/376233/articles/493274-study-on-thefretting-fatigue-behavior-of-alloy-zsgh4169-at-different-temperatures

\section{Hosted file}

figure3.pdf available at https://authorea.com/users/376233/articles/493274-study-on-thefretting-fatigue-behavior-of-alloy-zsgh4169-at-different-temperatures

\section{Hosted file}

figure4.pdf available at https://authorea.com/users/376233/articles/493274-study-on-thefretting-fatigue-behavior-of-alloy-zsgh4169-at-different-temperatures

\section{Hosted file}


figure5.pdf available at https://authorea.com/users/376233/articles/493274-study-on-thefretting-fatigue-behavior-of-alloy-zsgh4169-at-different-temperatures

\section{Hosted file}

figure6.pdf available at https://authorea.com/users/376233/articles/493274-study-on-thefretting-fatigue-behavior-of-alloy-zsgh4169-at-different-temperatures

\section{Hosted file}

figure7.pdf available at https://authorea.com/users/376233/articles/493274-study-on-thefretting-fatigue-behavior-of-alloy-zsgh4169-at-different-temperatures

\section{Hosted file}

figure8.pdf available at https://authorea.com/users/376233/articles/493274-study-on-thefretting-fatigue-behavior-of-alloy-zsgh4169-at-different-temperatures

\section{Hosted file}

figure9.pdf available at https://authorea.com/users/376233/articles/493274-study-on-thefretting-fatigue-behavior-of-alloy-zsgh4169-at-different-temperatures

\section{Hosted file}

figure10.pdf available at https://authorea.com/users/376233/articles/493274-study-on-thefretting-fatigue-behavior-of-alloy-zsgh4169-at-different-temperatures

\section{Hosted file}

figure11.pdf available at https://authorea.com/users/376233/articles/493274-study-on-thefretting-fatigue-behavior-of-alloy-zsgh4169-at-different-temperatures

\section{Hosted file}

figure12.pdf available at https://authorea.com/users/376233/articles/493274-study-on-thefretting-fatigue-behavior-of-alloy-zsgh4169-at-different-temperatures

\section{Hosted file}

figure13.pdf available at https://authorea.com/users/376233/articles/493274-study-on-thefretting-fatigue-behavior-of-alloy-zsgh4169-at-different-temperatures

\section{Hosted file}

figure14.pdf available at https://authorea.com/users/376233/articles/493274-study-on-thefretting-fatigue-behavior-of-alloy-zsgh4169-at-different-temperatures

\section{Hosted file}

figure15.pdf available at https://authorea.com/users/376233/articles/493274-study-on-thefretting-fatigue-behavior-of-alloy-zsgh4169-at-different-temperatures

\section{Hosted file}

figure16.pdf available at https://authorea.com/users/376233/articles/493274-study-on-thefretting-fatigue-behavior-of-alloy-zsgh4169-at-different-temperatures

\section{Hosted file}

figure17.pdf available at https://authorea.com/users/376233/articles/493274-study-on-thefretting-fatigue-behavior-of-alloy-zsgh4169-at-different-temperatures

\section{Hosted file}


table1.pdf available at https://authorea.com/users/376233/articles/493274-study-on-thefretting-fatigue-behavior-of-alloy-zsgh4169-at-different-temperatures

\section{Hosted file}

table2.pdf available at https://authorea.com/users/376233/articles/493274-study-on-thefretting-fatigue-behavior-of-alloy-zsgh4169-at-different-temperatures

\section{Hosted file}

table3.pdf available at https://authorea.com/users/376233/articles/493274-study-on-thefretting-fatigue-behavior-of-alloy-zsgh4169-at-different-temperatures

\section{Hosted file}

table4.pdf available at https://authorea.com/users/376233/articles/493274-study-on-thefretting-fatigue-behavior-of-alloy-zsgh4169-at-different-temperatures

\section{Hosted file}

table5.pdf available at https://authorea.com/users/376233/articles/493274-study-on-thefretting-fatigue-behavior-of-alloy-zsgh4169-at-different-temperatures 